\title{
LISSAJOUS KNOTS AND BILLIARD KNOTS
}

\author{
VAUGHAN F. R. JONES \\ Mathematics Department, University of California at Berkeley \\ Berkeley, California 94720, U.S.A. \\ E-mail:vfr@math.berkeley.edu \\ JÓZEF H. PRZYTYCKI \\ Department of Mathematics, The George Washington University \\ 2201 G St., Room 428 Funger Hall, Washington, D.C. 20052, U.S.A. \\ E-mail:przytyck@math.gwu.edu
}

\begin{abstract}
We show that Lissajous knots are equivalent to billiard knots in a cube. We consider also knots in general 3-dimensional billiard tables. We analyse symmetry of knots in billiard tables and show in particular that the Alexander polynomial of a Lissajous knot is a square modulo 2 .
\end{abstract}

0. Introduction. A Lissajous knot $K$ is a knot in $R^{3}$ given by the parametric equations

$$
x=\cos \left(\eta_{x} t+\phi_{x}\right), \quad y=\cos \left(\eta_{y} t+\phi_{y}\right), \quad z=\cos \left(\eta_{z} t+\phi_{z}\right),
$$

for integers $\eta_{x}, \eta_{y}, \eta_{z}$. A Lissajous link is a collection of disjoint Lissajous knots.

The fundamental question was asked in [BHJS94]: which knots are Lissajous?

One defines a billiard knot (or racquetball knot) as the trajectory inside a cube of a ball which leaves a wall at rational angles with respect to the natural frame, and travels in a straight line except for reflecting perfectly off the walls; generically it will miss the corners and edges, and will form a knot. We will show that these knots are precisely the same as the Lissajous knots. We will also speculate about more general billiard knots, e.g. taking another polyhedron instead of the ball, considering a non-Euclidean metric, or considering the trajectory of a ball in the configuration space of a flat billiard. We will illustrate these by various examples. For instance, the trefoil knot is not a Lissajous knot

1991 Mathematics Subject Classification: 57M25, 58F17.

This is an extended version of the talk given in August 1995, at the minisemester on Knot Theory at the Banach Center.

We would like to acknowledge the support from USAF grant 1-443964-22502.

The paper is in final form and no version of it will be published elsewhere. 
but we can easily realize it as a billiard knot in a room with a regular triangular floor $\left(^{1}\right)$; Fig. 0.1.

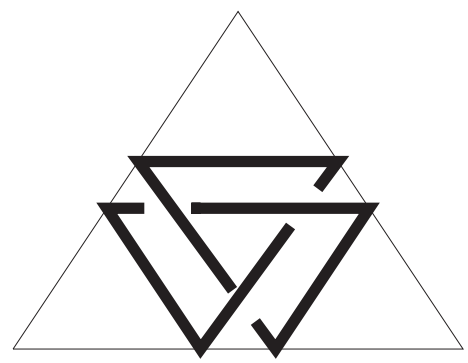

Fig. 0.1. The left handed trefoil knot in a room with a regular triangular floor ("Odin's triangle")

In the third part of the paper we discuss the symmetry of billiard knots. We sharpen the observation of [BHJS94] that a Lissajous knot is either strongly + amphicheiral or $Z_{2}$-periodic. We show that in the $Z_{2}$-periodic case the linking number of an axis of the $Z_{2}$ action with the knot is equal to \pm 1 . We use this to show that any Lissajous knot has an Alexander polynomial congruent to a square modulo 2. We also study other billiard knots which exibit symmetry (e.g. knots in a cylinder). Finally, in the fourth part, we consider knots given by trajectories which are not time-reversible. We are motivated by a movement in ponds of a unicellular slipper-shaped organism Paramecium Caudatum.

1. Deformation. We will consider here a family of curves which generalize Lissajous and billiard curves. We will show how to deform Lissajous knots to billiard knots inside this family.

Consider the family $\mathcal{F}$ of continuous fuctions $f: R \rightarrow R$ which satisfy the following properties:

(i) $f(t+1)=-f(t)$ (skew-period 1),

(ii) $f(-t)=f(t)$ (even function),

(iii) $f(0)=1$ (initial condition),

(iv) $f$ is strictly decreasing on the interval $\left(0, \frac{1}{2}\right)$.

As a consequence of our conditions we immediately have the following:

(v) $f$ has period $2 ; f(k)=(-1)^{k}$ for any integer $k$.

(vi) $f\left(\frac{1}{2}+t\right)=-f\left(\frac{1}{2}-t\right)$; i.e. $\left.f\left(\frac{1}{2}+t\right)\right)$ is an odd function, in particular $f\left(\frac{1}{2}\right)=0$.

(vii) $\mathcal{F}$ is in bijection with strictly decreasing functions from $\left[0, \frac{1}{2}\right]$ onto $[1,0]$.

(viii) $\mathcal{F}$ is a convex space; that is, if $f, g \in \mathcal{F}$, then $(1-s) f+s g$ is in $\mathcal{F}$ for any $s \in[0,1]$.

We show (vi) as an example: $f\left(\frac{1}{2}+t\right)=-f\left(-\frac{1}{2}+t\right)$ by (i) and $-f\left(-\frac{1}{2}+t\right)=-f\left(\frac{1}{2}-t\right)$ by (ii).

$\left(^{1}\right)$ This same figure was, according to [Cro95], used by the Norse people of Scandinavia and known as "Odin's triangle" or "Walknot". 
The simplest examples of our fuctions are $\cos (\pi t)$ and the piecewise linear "sawtooth" function $p(t)=2|| t\left|-2 E\left[\frac{1}{2}|t|\right]-1\right|-1$, where $E[x]$ is the greatest integer part of $x$; Fig. 1.1.

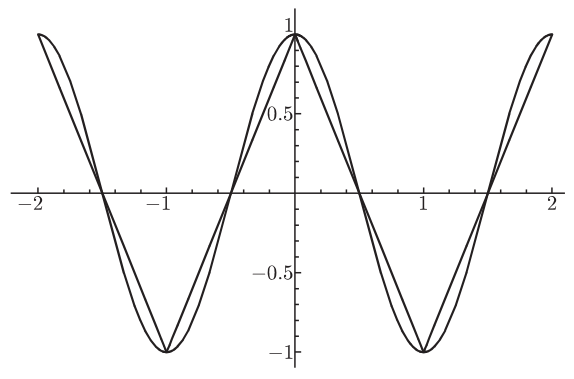

Fig. 1.1

Consider now any $f \in \mathcal{F}$.

LEMMA 1.1. Let $f_{1}(t)=f\left(n_{1} t+c_{1}\right), f_{2}(t)=f\left(n_{2} t+c_{2}\right)$, where $n_{1}, n_{2}$ are co-prime integers. Then the closed curve $\left(f_{1}(t), f_{2}(t)\right): R \rightarrow R^{2}$ has period 2 and one of the following conditions is satisfied:

(a) For some integers $k_{1}, k_{2}$,

$$
\frac{c_{2}}{n_{2}}-\frac{c_{1}}{n_{1}}=\frac{n_{1} k_{2}-n_{2} k_{1}}{n_{1} n_{2}}
$$

and our curve reaches two corners of the square $[-1,1] \times[-1,1]$ at $t_{0}=-\frac{c_{1}}{n_{1}}+\frac{k_{1}}{n_{1}}$ and $t_{0}+1$ (bounces off at these corners). The curve restricted to $\left[t_{0}, t_{0}+1\right]$ is locally embedded with $\left(n_{1}-1\right)\left(n_{2}-1\right) / 2$ double points.

(b) For $t \in[0,2]$ it describes a closed locally embedded curve with $2 n_{1} n_{2}-n_{1}-n_{2}$ double points for parameters $t^{\prime}, t^{\prime \prime}$ given by:

$$
\begin{aligned}
& t^{\prime}=\left(\frac{k_{2}}{n_{2}}+\frac{k_{1}}{n_{1}}\right)-\frac{c_{2}}{n_{2}} \quad \text { and } \quad t^{\prime \prime}=\left(\frac{k_{2}}{n_{2}}-\frac{k_{1}}{n_{1}}\right)-\frac{c_{2}}{n_{2}} \quad \text { and } \\
& t^{\prime}=\left(\frac{k_{2}}{n_{2}}+\frac{k_{1}}{n_{1}}\right)-\frac{c_{1}}{n_{1}} \quad \text { and } \quad t^{\prime \prime}=\left(-\frac{k_{2}}{n_{2}}+\frac{k_{1}}{n_{1}}\right)-\frac{c_{1}}{n_{1}} \text {. }
\end{aligned}
$$

Proof. $f\left(t_{1}\right)=f\left(t_{2}\right)$ iff $t_{1}= \pm t_{2}+2 k$. This follows from the fact that $f(t)$ is strictly increasing on the interval $[-1,0]$ and strictly decreasing on the interval $[0,1]$, symmetric with respect to the maximum and periodic. Therefore

$$
f_{1}\left(t^{\prime}\right)=f_{1}\left(t^{\prime \prime}\right) \quad \text { and } \quad f_{2}\left(t^{\prime}\right)=f_{2}\left(t^{\prime \prime}\right)
$$

reduces to

$$
n_{1} t^{\prime}+c_{1}= \pm n_{1}\left(t^{\prime \prime}+c_{1}\right)+2 k_{1} \text { and } n_{2} t^{\prime}+c_{2}= \pm n_{2}\left(t^{\prime \prime}+c_{2}\right)+2 k_{2} .
$$

Thus one has to consider four possibilities:

$$
n_{1} t^{\prime}+c_{1}=n_{1} t^{\prime \prime}+c_{1}+2 k_{1}, \quad n_{2} t^{\prime}+c_{2}=n_{2} t^{\prime \prime}+c_{2}+2 k_{2} .
$$

Therefore $t^{\prime}-t^{\prime \prime}=\frac{2 k_{1}}{n_{1}}=\frac{2 k_{2}}{n_{2}}$. Thus $2 k_{1} n_{2}=2 k_{2} n_{1}$ and because $n_{1}$ is relatively prime with respect to $n_{2}$, we have $k_{1}$ as a multiple of $n_{1}$ and $k_{2}$ of $n_{2}$. Finally $t^{\prime}-t^{\prime \prime}=2 m$ for 
some integer $m$, so our closed curve has no double points related to the equalities $(++)$. $(+-) \quad n_{1} t^{\prime}+c_{1}=n_{1} t^{\prime \prime}+c_{1}+2 k_{1}, \quad n_{2} t^{\prime}+c_{2}=-n_{2} t^{\prime \prime}-c_{2}+2 k_{2}$.

Thus $t^{\prime}=\left(\frac{k_{2}}{n_{2}}+\frac{k_{1}}{n_{1}}\right)-\frac{c_{2}}{n_{2}}$ and $t^{\prime \prime}=\left(\frac{k_{2}}{n_{2}}-\frac{k_{1}}{n_{1}}\right)-\frac{c_{2}}{n_{2}}$.

If $k_{1}$ is a multiple of $n_{1}$ then $t^{\prime} \equiv t^{\prime \prime} \bmod 2$ so we do not deal with a crossing. Thus to count all crossings we have to consider $k_{1} \in\left[1, n_{1}-1\right], k_{2} \in\left[0, n_{2}-1\right]$. These describe $\left(n_{1}-1\right) n_{2}$ crossings, and the crossings are all different since for pairs parametrizing crossings $\left(t_{1}^{\prime}, t_{1}^{\prime \prime}\right)$ and $\left(t_{2}^{\prime}, t_{2}^{\prime \prime}\right)$, one has to have $\left|t_{1}^{\prime}-t_{2}^{\prime}\right|<2$ and $t_{1}^{\prime} \neq t_{2}^{\prime \prime}$.

$(-+) \quad n_{1} t^{\prime}+c_{1}=-n_{1} t^{\prime \prime}-c_{1}+2 k_{1}, \quad n_{2} t^{\prime}+c_{2}=n_{2} t^{\prime \prime}+c_{2}+2 k_{2}$.

Thus $t^{\prime}=\left(\frac{k_{2}}{n_{2}}+\frac{k_{1}}{n_{1}}\right)-\frac{c_{1}}{n_{1}}$ and $t^{\prime \prime}=\left(-\frac{k_{2}}{n_{2}}+\frac{k_{1}}{n_{1}}\right)-\frac{c_{1}}{n_{1}}$. The consideration of $(+-)$ remains valid, only the roles of $f_{1}$ and $f_{2}$ are interchanged. Thus we get here $\left(n_{2}-1\right) n_{1}$ different crossings obtained for $t^{\prime}$ and $t^{\prime \prime}$ such that $k_{1} \in\left[0, n_{1}-1\right], k_{2} \in\left[1, n_{2}-1\right]$.

$(--) \quad n_{1} t^{\prime}+c_{1}=-n_{1} t^{\prime \prime}-c_{1}+2 k_{1}, \quad n_{2} t^{\prime}+c_{2}=-n_{2} t^{\prime \prime}-c_{2}+2 k_{2}$.

Thus $n_{1} c_{2}-n_{2} c_{1}=n_{1} k_{2}-n_{2} k_{1}$, or equivalently $\frac{c_{2}}{n_{2}}-\frac{c_{1}}{n_{1}}=\frac{k_{2}}{n_{2}}-\frac{k_{1}}{n_{1}}$. This corresponds to case (a) of Lemma 1.1. One can comment on this condition:

First of all, it is independent of $t$; thus for any $t^{\prime}$ there is a unique (up to period 2) $t^{\prime \prime}$ $\left(t^{\prime \prime}=-t^{\prime}-\frac{2 c_{1}}{n_{1}}+\frac{2 k_{1}}{n_{1}}\right)$ such that $f_{1}\left(t^{\prime}\right)=f_{1}\left(t^{\prime \prime}\right)$, and we can say that our curve "bends on itself" (see Fig. 1.2). To see this clearly, notice that our curve reaches two corners of

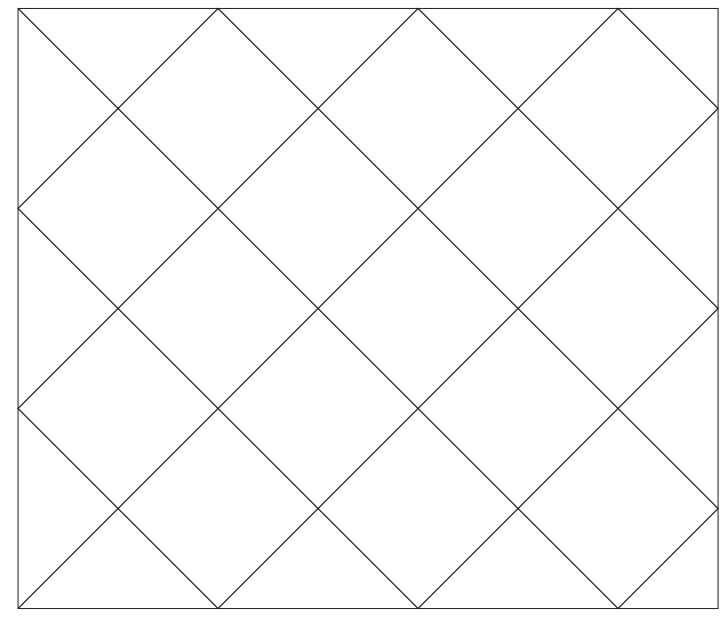

Fig. 1.2. The curve $(f(6 t+1), f(7))$ bounces at corners and bends on itself.

the square $[-1,1] \times[-1,1]$, namely reparametrize our curve $\left(f_{1}(t), f_{2}(t)\right)$ by changing $t$ to $t-\frac{c_{1}}{n_{1}}+\frac{k_{1}}{n_{1}}$ (which by above is the same as changing $t$ to $t-\frac{c_{2}}{n_{2}}+\frac{k_{2}}{n_{2}}$ ). In the new parametrization we get the curve $\left(f\left(n_{1} t+k_{1}\right), f\left(n_{2} t+k_{2}\right)\right)$, so it reaches corners at $t=0$ and $t=1$, then bounces at these corners and "bends on itself" (by condition (ii) and the fact that $f(1+t)=f(1-t))$. In particular if $n_{1}$ and $n_{2}$ are odd then the curve goes through 0 .

To finish the proof of Lemma 1.1 we have to analyse when crossings described in the $(+-)$ and $(-+)$ cases are different. 
Let

and

$$
\left(t_{1}^{\prime}, t_{1}^{\prime \prime}\right)=\left(\left(\frac{k_{2}}{n_{2}}+\frac{k_{1}}{n_{1}}\right)-\frac{c_{2}}{n_{2}},\left(\frac{k_{2}}{n_{2}}-\frac{k_{1}}{n_{1}}\right)-\frac{c_{2}}{n_{2}}\right)
$$

$$
\left(t_{2}^{\prime}, t_{2}^{\prime \prime}\right)=\left(\left(\frac{k_{2}^{\prime}}{n_{2}}+\frac{k_{1}^{\prime}}{n_{1}}\right)-\frac{c_{1}}{n_{1}},\left(-\frac{k_{2}^{\prime}}{n_{2}}+\frac{k_{1}^{\prime}}{n_{1}}\right)-\frac{c_{1}}{n_{1}}\right)
$$

be parameters giving crossings in $(+-)$ and $(-+)$, respectively. Thus $t_{1}^{\prime}=t_{2}^{\prime}$ or $t_{1}^{\prime \prime}=t_{2}^{\prime}$ if and only if

$$
\frac{c_{2}}{n_{2}}-\frac{c_{1}}{n_{1}}=\frac{k_{2}-k_{2}^{\prime}}{n_{2}}-\frac{k_{1}^{\prime} \pm k_{1}}{n_{1}} .
$$

This is exactly the (a) case of Lemma 1.1 and it was analysed in case (--); that is, a pair of crossings coincide because our curve "bends on itself".

If the crossings of cases $(+-)$ and $(-+)$ are different, we have $\left(n_{1}-1\right) n_{2}+\left(n_{2}-1\right) n_{1}=$ $2 n_{1} n_{2}-n_{1}-n_{2}$ of crossings. This completes the proof of Lemma 1.1.

Let $f \in \mathcal{F}$ and consider a closed curve in a 3 -dimensional cube, parametrized by $F(t)=\left(f_{1}(t), f_{2}(t), f_{3}(t)\right)$, where $f_{i}(t)=f\left(n_{i} t+c_{i}\right)$. Of course, our curve has period 2 .

LEMMA 1.2. $F(t)$, for $t \in[0,2]$, parametrizes a simple closed curve (a knot) unless

(i) $\frac{c_{2}}{n_{2}}-\frac{c_{1}}{n_{1}}=\frac{m}{n_{1} n_{2}}$, or

(ii) $\frac{c_{3}}{n_{3}}-\frac{c_{1}}{n_{1}}=\frac{m^{\prime}}{n_{1} n_{3}}$, or

(iii) $\frac{c_{3}}{n_{3}}-\frac{c_{2}}{n_{2}}=\frac{m^{\prime \prime}}{n_{2} n_{3}}$ for some integer $m$ (or $m^{\prime}$ or $\left.m^{\prime \prime}\right)$.

Furthermore if (i) (resp. (ii) or (iii)) holds but not all three, then we deal with a singular knot with $n_{3}-1$ (resp. $n_{2}-1$ or $\left.n_{1}-1\right)$ double points.

Proof. We want to find all possible self-crossings of the curve $F$ (on $[-1,1]$ ), so we have to solve the system of equations

$$
f_{1}\left(t^{\prime}\right)=f_{1}\left(t^{\prime \prime}\right), \quad f_{2}\left(t^{\prime}\right)=f_{2}\left(t^{\prime \prime}\right), \quad f_{3}\left(t^{\prime}\right)=f_{3}\left(t^{\prime \prime}\right),
$$

or equivalently

$n_{1} t^{\prime}+c_{1}= \pm n_{1} t^{\prime \prime}+c_{1}+2 k_{1}, \quad n_{2} t^{\prime}+c_{2}= \pm n_{2} t^{\prime \prime}+c_{2}+2 k_{2}, \quad n_{3} t^{\prime}+c_{3}= \pm n_{3} t^{\prime \prime}+c_{3}+2 k_{3}$.

We have to consider 8 possibilities, which we can denote succinctly as $\left(\varepsilon_{1}, \varepsilon_{2}, \varepsilon_{3}\right)$ where $\varepsilon_{i}= \pm$. We base our analysis on Lemma 1.1 and its proof.

$(++\varepsilon)$ If two $\varepsilon$ 's are + , say $\varepsilon_{1}, \varepsilon_{2}$, then by case $(++)$ of the proof of Lemma 1.1, we will not produce a self-crossing.

$(---)$ From case $(--)$ of the proof of Lemma 1.1 applied to any pair $f_{i}, f_{j}$, we get the system of equations

$$
\frac{c_{2}}{n_{2}}-\frac{c_{1}}{n_{1}}=\frac{k_{2}}{n_{2}}-\frac{k_{1}}{n_{1}}, \quad \frac{c_{3}}{n_{3}}-\frac{c_{2}}{n_{2}}=\frac{k_{3}}{n_{3}}-\frac{k_{2}}{n_{2}}, \quad \frac{c_{1}}{n_{1}}-\frac{c_{3}}{n_{3}}=\frac{k_{1}}{n_{1}}-\frac{k_{3}}{n_{3}} ;
$$

of course, only two of these are independent. Observe, as in case (--), that by reparametrizing the curve by a shift $t \rightarrow\left(t-\frac{c_{1}}{n_{1}}-\frac{k_{1}}{n_{1}}\right)$, one gets the curve $\left(f\left(n_{1} t-k_{1}\right)\right.$, $\left.f\left(n_{2} t-k_{2}\right), f\left(n_{3} t-k_{3}\right)\right)$ and that this curve hits corners at $t=0$ and $t=1$ then bounces at these corners and "bends on itself". 
$(--+)$ If two $\varepsilon$ 's are - , say $\varepsilon_{1}, \varepsilon_{2}$, then by case $(--)$ of the proof of Lemma 1.1,

$$
\frac{c_{2}}{n_{2}}-\frac{c_{1}}{n_{1}}=\frac{k_{2}}{n_{2}}-\frac{k_{1}}{n_{1}}, \quad t^{\prime}=-t^{\prime \prime}-\frac{2 c_{1}}{n_{1}}+\frac{2 k_{1}}{n_{1}}
$$

and by case $(-+)$ applied to $\left(f_{1}, f_{3}\right)$ and $\left(f_{2}, f_{3}\right)$,

$$
\begin{aligned}
& t^{\prime}=\left(\frac{k_{3}}{n_{3}}+\frac{k_{1}}{n_{1}}\right)-\frac{c_{1}}{n_{1}} \quad \text { and } \quad t^{\prime \prime}=\left(-\frac{k_{3}}{n_{3}}+\frac{k_{1}}{n_{1}}\right)-\frac{c_{1}}{n_{1}}, \quad \text { and } \\
& t^{\prime}=\left(\frac{k_{3}}{n_{3}}+\frac{k_{2}}{n_{2}}\right)-\frac{c_{2}}{n_{2}} \quad \text { and } \quad t^{\prime \prime}=\left(-\frac{k_{3}}{n_{3}}+\frac{k_{2}}{n_{2}}\right)-\frac{c_{2}}{n_{2}} .
\end{aligned}
$$

From the first and the second conditions, one gets

$$
t^{\prime}=\frac{k_{3}}{n_{3}}+\frac{k_{1}}{n_{1}}-\frac{c_{1}}{n_{1}}
$$

$k_{1}$ and $k_{2}$ are determined, so the only choice we have is for $k_{3}$, thus we get $k_{3}-1$ double points. Assuming that (---) cannot be satisfied, these are the only double points of our closed curve.

This completes the proof of Lemma 1.2.

THEOREM 1.3. For given integers $n_{1}, n_{2}, n_{3}$ and real numbers $c_{1}, c_{2}, c_{3}$ the knot (up to ambient isotopy) $F(t)=\left(f_{1}(t), f_{2}(t), f_{3}(t)\right)$, where $f_{i}(t)=f\left(n_{i} t+c_{i}\right)$, does not depend on the choice of $f \in \mathcal{F}$. This theorem also holds for the case of a singular knot.

Proof. Let $f_{0}, f_{1} \in \mathcal{F}$. Consider the homotopy between $f_{0}$ and $f_{1}: f_{s}=(1-s) f_{0}+$ $s f_{1}$. By convexity of $\mathcal{F}, f_{s} \in \mathcal{F}$. Thus we have also the homotopy $F_{s}=(1-s) F_{0}+$ $s F_{1}$ between $F_{0}(t)=\left(\left(f_{0}\right)_{1}(t),\left(f_{0}\right)_{2}(t),\left(f_{0}\right)_{3}(t)\right)$ and $F_{1}(t)=\left(\left(f_{1}\right)_{1}(t),\left(f_{1}\right)_{2}(t),\left(f_{1}\right)_{3}(t)\right)$. Furthermore if $F_{0}$ is a knot, $F_{t}$ is also a knot and thus represents the same topological type. Similarly, if $F_{0}$ is a singular knot, then $F_{s}$ has double points for the same parameters $t$ as $F_{0}$ for any $s$ (by Lemma 1.1). The proof of Theorem 1.3 is complete.

COROLlary 1.4. Lissajous and billiard knots are the same up to ambient isotopy.

2. General billiards. There is extensive literature devoted to billiards, including 3-dimensional billiard tables [GKT94, Ta95]. The problem of closed trajectories has also been widely studied [BGKT94, GSV92, KMS86, Ta95]. Apparently, however, the problem of knots as trajectories of a ball in a billiard table has not been studied before $\left(^{2}\right)$.

We give, in this section, some examples of knots as billiard trajectories and formulate a few conjectures, but the field is widely open for future research; and as a new field, it has plenty of possible directions and problems (from easy to very difficult ones).

We start from the general definition.

Definition 2.1 ([Ta95]). A billiard table (or racquetball room) is a Riemannian manifold $M$ with a piecewise smooth boundary. The billiard dynamical system in $M$ is generated by the free motion of a mass-point (called a billiard ball) subject to elastic reflection off the boundary. This means that the point moves along a geodesic line in $M$ with a constant speed until it hits the boundary. At a smooth boundary point the billiard

\footnotetext{
$\left({ }^{2}\right)$ For us the initial motivation was the possiblity of the hand drawing of Lissajous knots.
} 
ball reflects so that the tangential component of its velocity remains the same, while the normal component changes its sign. If the billiard ball hits a corner, its further motion is usually not defined (for us the important exception is a right dihedral angle at an edge and a cubic corner in the polyhedron in $R^{3}$ ).

A billiard knot (or link) is a simple closed trajectory (trajectories) of a ball in a 3-dimensional billiard table. $\left({ }^{3}\right)$ The simplest billiards to consider would be polytopes (finite convex polyhedra in $R^{3}$ ). But even for Platonian bodies we know nothing of the knots they support except in the case of the cube. It seems that polytopes which are the products of polygons and the interval $([-1,1])$ (i.e. polygonal prisms) are more accessible. This is the case because diagrams of knots are billiard trajectories in 2-dimensional tables. We will list some examples below.

EXAMPLE 2.2. (i) The trivial knot and the trefoil knot are the trajectories of a ball in a room (prism) with an acute triangular floor. In Fig. 2.1(a), the diagram of the trivial knot is an inscribed triangle $\Delta_{I}$ whose vertices are the feet of the triangle's altitudes. If we move the first vertex of $\Delta_{I}$ slightly, each of its edges splits into two and we get the diagram of the trefoil. We should be careful with the altitude of the trajectory: We start from level 1 at the vertex close to the vertex of $\Delta_{I}$ and opposite to the shortest edge of $\Delta_{I}$. Then we choose the vertical parameter so that the trajectory has 3 maxima and three minima (Fig. 2.1(b)).

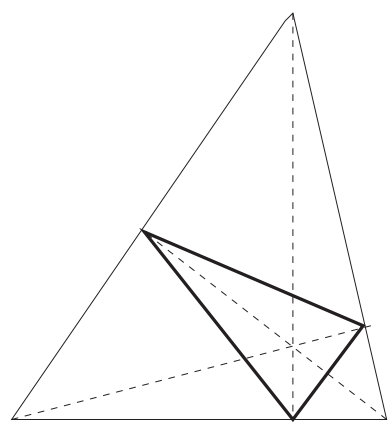

(a)

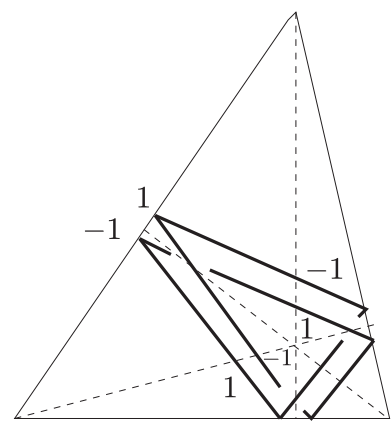

(b) The trefoil knot as a trajectory of a ball in a room whose floor is an acute triangle

Fig. 2.1

(ii) The trivial knot is a trajectory of a ball in a room with a right triangular floor, Fig. 2.2.

(iii) If the floor of a room is a general obtuse triangle, it is an open problem whether any knot can be realized as the trajectory of a ball in it. However we have the general theorem that periodic points are dense (in the phase space of the billiard flow) in a rational polygon (that is, all polygonal angles are rational with respect to $\pi$ ) [BGKT94].

$\left({ }^{3}\right)$ One can also consider closed trajectories in the phase space of a 2-dimensional billiard table but we will not pursue this possibility here. 


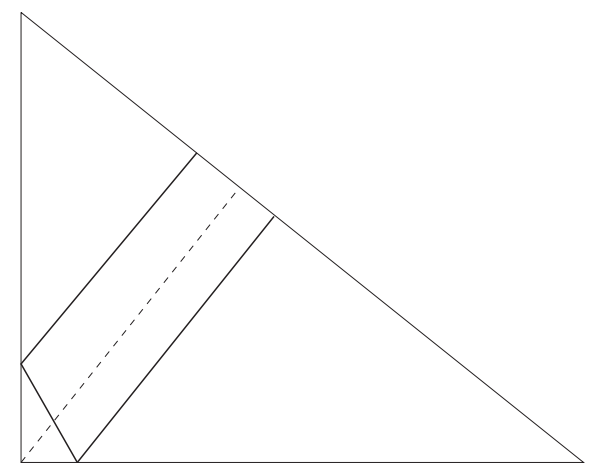

Fig. 2.2. The trivial knot can be realized as a trajectory of a ball in any room with a right triangular floor.

Example 2.2(i) is of interest because it was shown in [BHJS94] that the trefoil knot is not a Lissajous knot and thus it is not a trajectory of a ball in a room with a rectangular floor. More generally we show in Section 3 that no nontrivial torus knot is a Lissajous knot. However, we can construct infinitely many torus knots in prisms and in the cylinder.

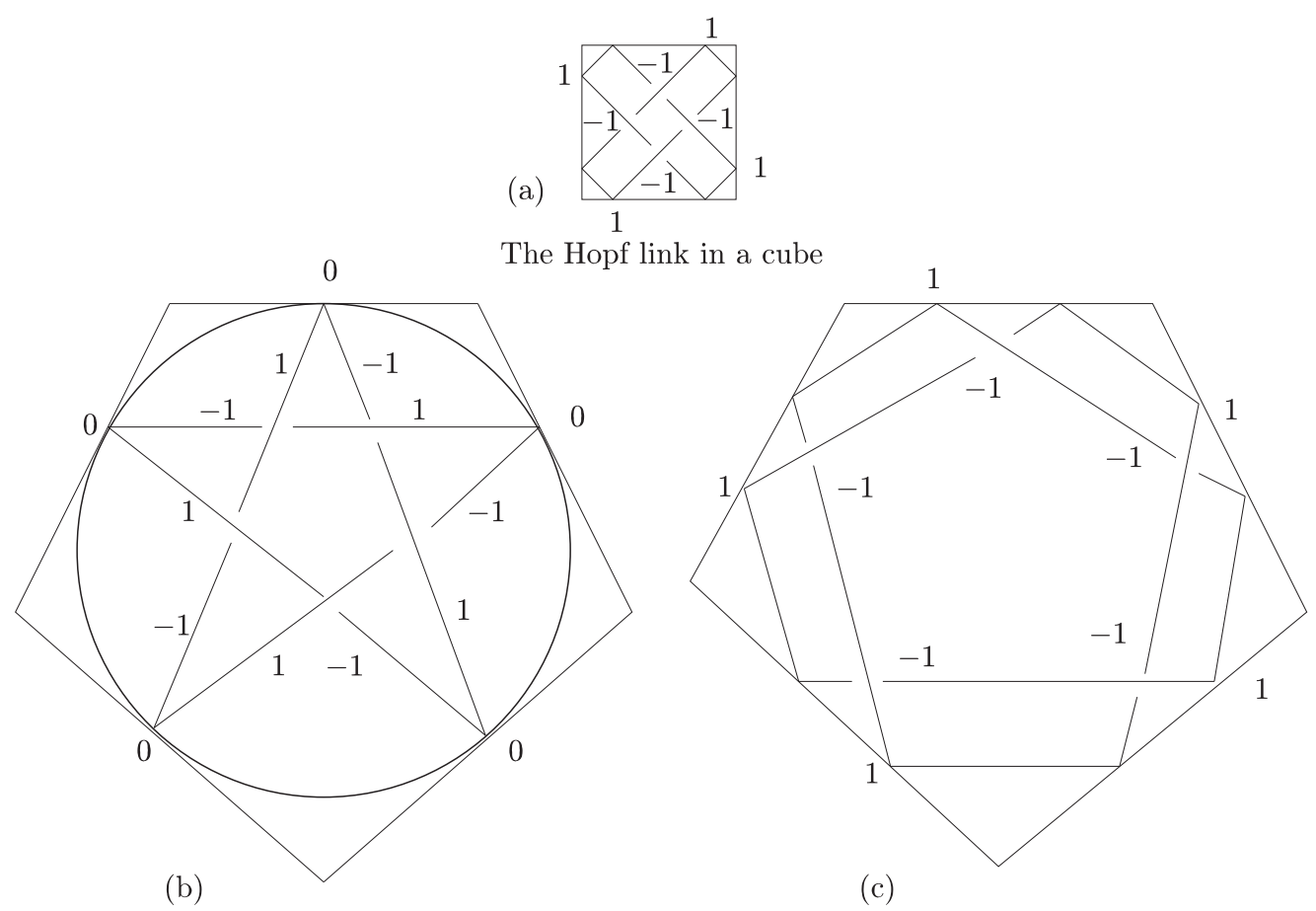

The torus knot of type $(5,2)$ as a trajectory of a ball in a room with a regular pentagonal floor

Fig. 2.3 
ExAmple 2.3. (i) Any torus knot (or link) of type $(n, 2)$ can be realized as a trajectory of a ball in a room whose floor is a regular $n$-gon $(n \geq 3)$. Fig. 0.1 shows the $(3,2)$ torus knot (trefoil) in the regular triangular prism; Fig. 2.3(a) depicts the $(4,2)$ torus link in the cube; and Fig. 2.3(b)(c) illustrates the $(5,2)$ torus knot in a room with a regular pentagonal floor.

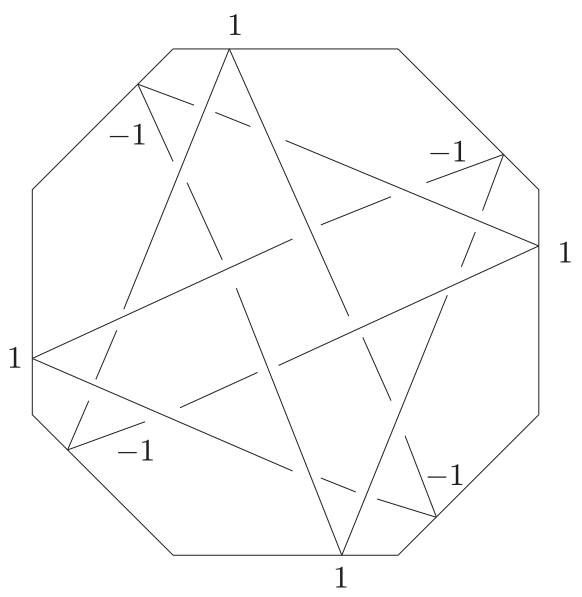

(a) The torus knot of type $(4,3)$ as a trajectory of a ball in a room with a regular octagonal floor

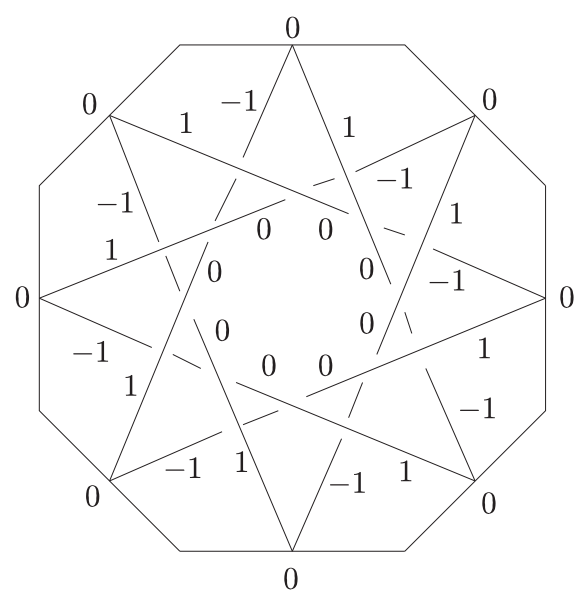

(b) The torus knot of type $(8,3)$ as a trajectory of a ball in a room with a regular octagonal floor

Fig. 2.4

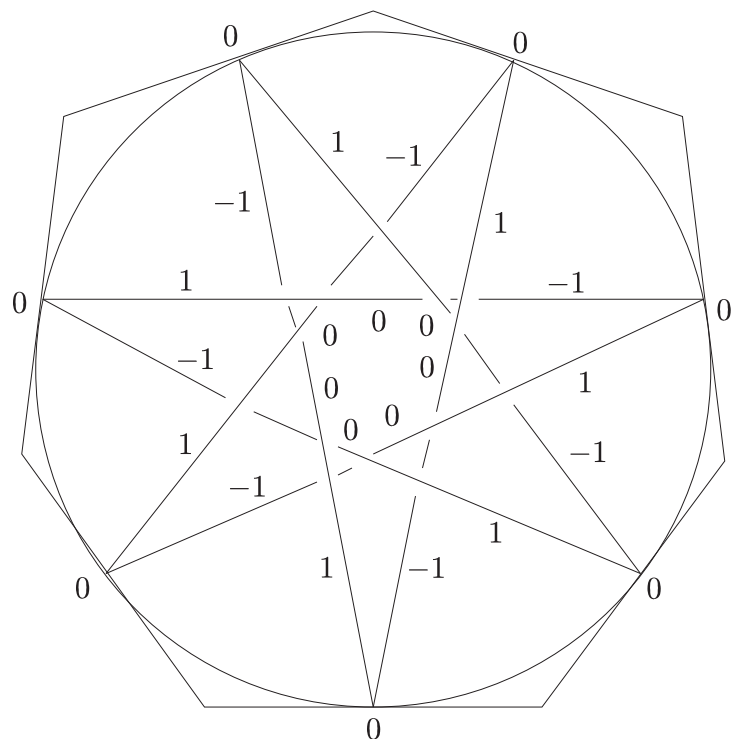

Fig. 2.5. The torus knot of type $(7,3)$ realized as a trajectory of a ball in a room with a regular heptagonal floor. 
(ii) The $(4,3)$ torus knot is a trajectory of a ball in a room with regular octagonal floor; Fig. 2.4(a).

(iii) Figures 2.4(b) and 2.5 illustrate how to construct a torus knot (or link) of type $(n, 3)$ in a room with a regular $n$-gonal floor for $n \geq 7$.

(iv) Any torus knot (or link) of type $(n, k)$, where $n \geq 2 k+1$, can be realized as a trajectory of a ball in a room with a regular $n$-gonal floor. The pattern generalizes that of Figures 2.3(b), 2.4(b) and 2.5. Edges of the diagram go from the center of the $i$ th edge to the center of the $(i+k)$ th edge of the $n$-gon. The ball bounces from walls at altitude 0 and its trajectory has $n$ maxima and $n$ minima. The whole knot (or link) is $Z_{n}$-periodic.

EXAMPLE 2.4. Let $D$ be a closed billiard trajectory on a 2-dimensional polygonal table. If $D$ is composed of an odd number of segments, then we can always find the "double cover" closed trajectory $D^{(2)}$ in the neighborhood of $D$ (each segment will be replaced by two parallel segments on the opposite sides of the initial segment). This idea can be used to construct, for a given billiard knot $K$ in a polygonal prism (the projection $D$ of $K$ having an odd number of segments), a 2-cable $K^{(2)}$ of $K$ as a billiard trajectory (with projection $D^{(2)}$ ). This idea is illustrated in Fig. 2.1 and 2.3(c) (the $(5,2)$ torus knot as a 2-cable of a trivial one). Starting from Example 2.3(iv) we can construct a 2-cable of a torus knot of the type $(n, k)$ in a regular $n$-gonal prism, for $n$ odd and $n \geq 2 k+1$.

It follows from [BHJS94] that 3-braid alternating knots of the form $\left(\sigma_{1} \sigma_{2}^{-1}\right)^{2 k}$ are not Lissajous knots as they have a non-zero Arf invariant (see Section 3). For $k=1$ we have the figure eight knot and for $k=2$ the $8_{18}$ knot [Ro76].

EXAMPLE 2.5. (i) The Listing knot (figure eight knot) can be realized as a trajectory of a ball in a room with a regular octagonal floor, Fig. $2.6\left(^{4}\right)$.
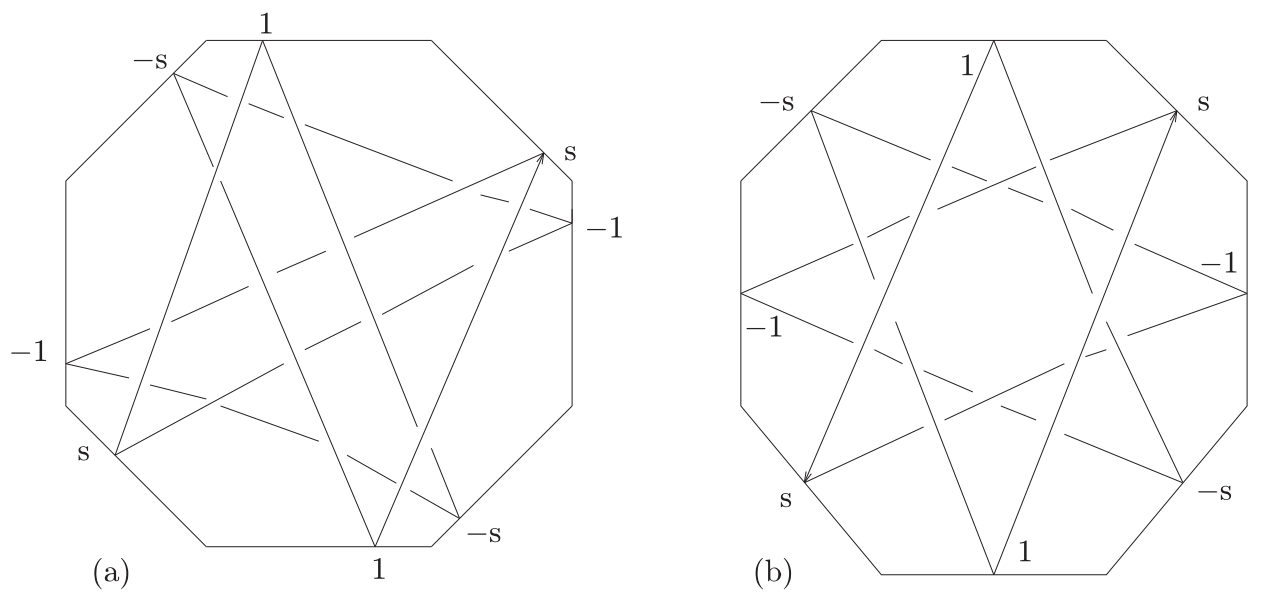

Fig. 2.6. The Listing (figure eight) knot as a trajectory of a ball in a room with a regular octagonal floor.

$\left({ }^{4}\right)$ This drawing was motivated by R. Randell's drawing at his talk at Banach Center in August 1995 and ascribed to E. Flapan, M. Meissen and J. Van Buskirk. 
(ii) Fig. 2.7 describes the knot $8_{18}$ as a trajectory of a ball in a room with a regular octagonal floor. This pattern can be extended to obtain the knot (or link) which is the closure of the three braid $\left(\sigma_{1} \sigma_{2}^{-1}\right)^{2 k}$ in a regular $4 k$-gonal prism $(k>1)$.

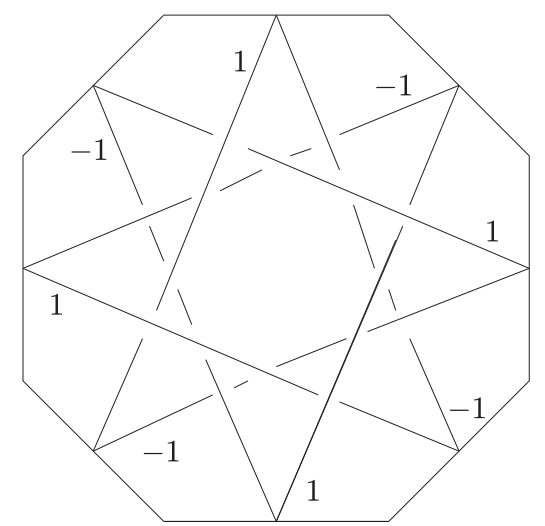

Fig. 2.7. The knot $8_{18}$ [Ro76], realized as a trajectory of a ball in a room with a regular octagonal floor.

We do not know if any polytope will support an infinite number of different knot types, however such is the case for the cylinder $D^{2} \times[-1,1]$.

ExAmple 2.6. (i) Any torus knot (or link) of type $(n, k)$, where $n \geq 2 k+1$, can be realized as a trajectory of a ball in the cylinder; compare Fig. 2.3(b), Fig. 2.4(b) and Fig. 2.5.

(ii) Every knot (or link) which is the closure of the three braid $\left(\sigma_{1} \sigma_{2}^{-1}\right)^{2 k}$ can be realized as the trajectory of a ball in the cylinder. See Fig. 2.6(b) for the case of $k=1$ (Listing knot) and Fig. 2.7 for the case of $k=2$ and the general pattern.

Any type of knot can be obtained as a trajectory of a ball in some polyhedral billiard (possibly very complicated). To see this, consider a polygonal knot in $R^{3}$ and place "mirrors" (walls) at any vertex, in such a way that the polygon is a "light ray" (ball) trajectory.

Conjecture 2.7. Any knot type can be realized as the trajectory of a ball in a polytope.

Conjecture 2.8. Any polytope supports an infinite number of different knot types.

Problem 2.9. 1. Is there a convex polyhedral billiard in which any knot type can be realized as the trajectory of a ball?

2. Can any knot type be realized as the trajectory of a ball in a room with a regular polygonal floor?

3. Which knot types can be realized as trajectories of a ball in a cylinder $\left(D^{2} \times[-1,1]\right)$ ?

3. Symmetry of billiard knots. Until now we have been unable to classify all billiard knots in any nontrivial 3-dimensional billiard (trivial being for example $D^{3}$ which has only the trivial knot as a trajectory). We can, however, exclude some knot types as 
closed trajectories in some billiards, due to symmetry principles. This was first observed for Lissajous knots in [BHJS94]. Namely, let $f \in \mathcal{F}$ (see Section 1), and $f_{i}(t)=f\left(n_{i} t+c_{i}\right)$, $i=1,2,3$. We can assume that $n_{1}, n_{2}$ are odd. We call the knot described by $\left(f_{1}, f_{2}, f_{3}\right)$ even if $n_{3}$ is even and odd otherwise.

Definition 3.1. (i) A knot in $R^{3}$ is called strongly + amphicheiral if it has a realization in $R^{3}$ which is preserved by a central symmetry $((x, y, z) \rightarrow(-x,-y,-z))$ (this symmetry changes orientation of $\left.R^{3}\right)$. "Plus" means that the involution preserves the orientation of the knot.

(ii) A knot is called $n$-periodic if there exists an action of $Z_{n}$ on $S^{3}$ which preserves the knot and the set of fixed points of the action is a circle disjoint from the knot.

Because $f(t+1)=-f(t)$ we have

THEOREM 3.2. An even Lissajous knot is $Z_{2}$-periodic and an odd Lissajous knot is strongly + amphicheiral.

We will strengthen the above theorem by showing the following:

THEOREM 3.3. In the even case the linking number of the axis of the $Z_{2}$-action with the knot is equal to \pm 1 .

Sketch of proof $\left(^{5}\right)$. Consider $f_{1}, f_{2}$ defined as before $\left(n_{1}, n_{2}\right.$ are co-prime odd numbers).

LEMMA 3.4. $\left(f_{1}, f_{2}\right)(t)$ goes through 0 if and only if

$$
\frac{c_{2}}{n_{2}}-\frac{c_{1}}{n_{1}}=\frac{k}{n_{1} n_{2}} \quad \text { for some integer } k .
$$

Proof. (i) If $\left(f_{1}, f_{2}\right)(t)$ goes through 0 , then for some integers $h_{1}, h_{2}$,

$$
n_{1} t+c_{1}=\frac{1}{2}+h_{1}, \quad n_{2} t+c_{2}=\frac{1}{2}+h_{2}
$$

thus

Finally

$$
t=-\frac{c_{1}}{n_{1}}+\frac{1}{2 n_{1}}+\frac{h_{1}}{n_{1}}=-\frac{c_{2}}{n_{2}}+\frac{1}{2 n_{2}}+\frac{h_{2}}{n_{2}} .
$$

for some integer $k$.

$$
\frac{c_{2}}{n_{2}}-\frac{c_{1}}{n_{1}}=\frac{h_{2} n_{1}-h_{1} n_{2}}{n_{1} n_{2}}+\frac{n_{1}-n_{2}}{2 n_{1} n_{2}}=\frac{k}{n_{1} n_{2}}
$$

(ii) If

$$
\frac{c_{2}}{n_{2}}-\frac{c_{1}}{n_{1}}=\frac{k}{n_{1} n_{2}}
$$

then we can find $h_{1}$ and $h_{2}$ such that $k=h_{2} n_{1}-h_{1} n_{2}+\frac{1}{2}\left(n_{1}-n_{2}\right)$. Then, if we choose $t$ such that $n_{1} t+c_{1}=\frac{1}{2}+h_{1}$, then $n_{2} t+c_{2}=\frac{1}{2}+h_{2}$. For this $t, f_{1}(t)=f_{2}(t)=0$.

LEMMA 3.5. If $\left(f_{1}, f_{2}\right)$ does not go through zero then there is a well defined linking number, $l k\left(f_{1}, f_{2}\right)$, of the curve with 0 , and it is equal to +1 or -1 .

$\left({ }^{5}\right)$ The detailed proof will be given elsewhere; see [Pr95]. 
Proof. The idea of the proof is as follows: We show that for a generic $c_{i}$ the assumption of the theorem holds; and in the degenerate case, when the curve goes through 0 , it also reaches antipodal corners of the square at which it bounces and "bends on itself". A small deformation of the parameters $c_{i}$ naturally gives the linking number \pm 1 and any deformation omitting 0 does not change this linking number.

More precisely: As proven in Section 1, the case of $\frac{c_{2}}{n_{2}}-\frac{c_{1}}{n_{1}}=\frac{k}{n_{1} n_{2}}$ is exactly the case when the curve reaches two antipodal corners of the square $[-1,1] \times[-1,1]$, bounces off at these corners and "bends on itself". In all other cases the curve $\left(f_{1}, f_{2}\right)(t)$ has $2 n_{1} n_{2}-n_{1}-n_{2}$ double points and is embedded outside double points.

For simplicity (e.g. for better visualization of the curve but without loss of generality) let us consider the case of billiard curves. Then $\left(f_{1}, f_{2}\right)(t)$ is a piece-wise linear curve without horizontal segments. We can visualize the linking number by drawing an interval from $(0,0)$ to $(0,1)$ and counting signed intersections of the interval with segments of the billiard trajectory. $\left(f_{1}, f_{2}\right)$ is a continuous function with respect to parameters $c_{i}$. Fix $c_{1}$ (we can assume $c_{1}=0$ ) and consider a curve $\left(f_{1}, f_{2}^{\prime}\right)$ with parameter $c_{2}^{\prime}=c_{2}+\epsilon$ for small $\epsilon$. When we move from $\epsilon=0$ to $\epsilon$ close to (but different than) zero, then every segment splits into two segments symmetric with respect to $(0,0)$ (we also create new segments close to corners where the curve was "bent on itself"). With the exception of the segments which arise from the one going through $(0,0)$ all other split segments will cancel their contributions to the linking number. Thus the linking number will be \pm 1 . Any further deformation omitting $(0,0)$ will preserve the linking number.

COROLlary 3.6. The Alexander polynomial of every Lissajous knot is a square modulo 2 .

Pr o of. For a strongly + amphicheiral knot the Alexander polynomial is a square by Hartley and Kawauchi [HK79]. For $Z_{2}$-periodic knots we can use the following theorem of Murasugi (assuming $k= \pm 1$ ).

THEOREM 3.7 ([Mu71]). Let L be an r-periodic oriented link with linking number $k$ with the fixed point set axis. Then

$$
\Delta_{L}(t) \equiv \Delta_{L_{*}}^{r}(t)\left(1+t+t^{2}+\ldots+t^{|k|-1}\right)^{r-1} \bmod r
$$

where $L_{*}=L / Z_{r}$ and $r$ is a prime number.

COROLlary 3.8. (i) ([BHJS94]) The Arf invariant of the Lissajous knot is 0.

(ii) A nontrivial torus knot is not a Lissajous knot.

(iii) For $\eta_{z}=2$ a Lissajous knot is a two bridge-knot and its Alexander polynomial is congruent to 1 modulo 2 .

Proof. (i) It follows from Corollary 3.6 and the fact that the Arf invariant is, modulo 2, the first nontrivial coefficient of the Conway-Alexander polynomial.

(ii) The Alexander polynomial of a nontrivial torus knot is not a square modulo 2.

(iii) A Lissajous knot with $\eta_{z}=2$ has two maxima so it is a two-bridge knot. If we divide it by the $Z_{2}$-action we get a one-bridge knot as an orbit (thus trivial knot). Finally, we use the Murasugi theorem $\left(\Delta_{L_{*}}=1\right)$.

Motivated by the case of $Z_{2}$-periodic knots we propose 
CONJeCture 3.9. Turks head knots (e.g. the closure of the 3-string braids $\left(s_{1} \bar{s}_{2}\right)^{2 k+1}$ ) are not Lissajous. (Observe that they are strongly + amphicheiral.)

We do not think, as the above conjecture shows, that the converse to Theorem 3.2 holds. However for $Z_{2}$-periodic knots it may hold (the method sketched in Section 0.4 of [BHJS94] may work).

Problem 3.10. Let $K$ be a $Z_{2}$-periodic knot, such that the linking number of the axis of the $Z_{2}$-action with $K$ is equal to \pm 1 . Is $K$ an even Lissajous knot?

The first prime knots (in the knot tables [Ro76]) which may or may not be Lissajous are $7_{5}, 8_{3}, 8_{6}$.

We can divide billiard knots in the cylinder into two classes, even and odd, depending on the number of segments in the projection into the base. Let $f_{1,2}(t)$ parametrize the projection of the knot into the base, and $f_{3}(t)$ describe the vertical direction, where $t \in[-1,1]$. We have $f_{1,2}\left(t+\frac{2}{n}\right)=e^{2 \pi \frac{k}{n}} f_{1,2}(t)$, where $n>2 k$; and $f_{3}\left(t+\frac{1}{m}\right)=-f_{3}(t)$. $n$ is the number of segments in the projection of the knot into the base and $m$ is the number of maxima of the trajectory of $K$.

THEOREM 3.11. An even billiard knot in a cylinder is either $Z_{2}$-periodic or strongly + amphicheiral, depending on whether it has an even or odd number of maxima. In the $Z_{2}$-periodic case the linking number of an axis of the $Z_{2}$ action with the knot is equal to $\pm k$.

Proof. For even $n$ one has $f_{1,2}(t+1)=-f_{1,2}(t)$ and $f_{3}(t+1)=f_{3}(t)$ or $-f_{3}(t)$ depending on whether the knot has an even or odd number of maxima; $t$ is considered modulo 2. The value of the linking number is immediately visible from the diagram (compare Fig. 2.4(b) for the case $n=8, k=3$ ). Thus Theorem 3.11 follows.

A similar result also holds for an isosceles right triangular prism.

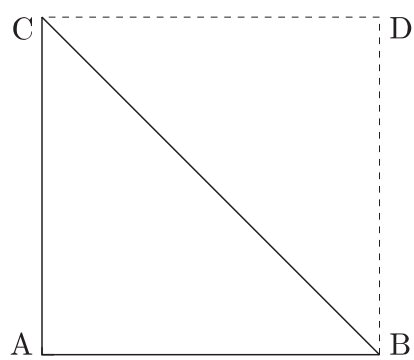

(a)

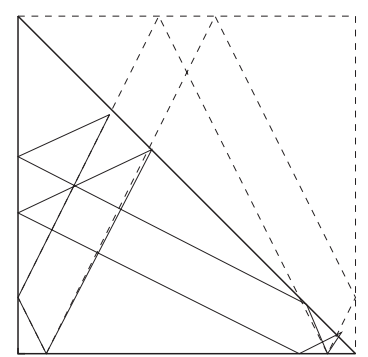

(b)

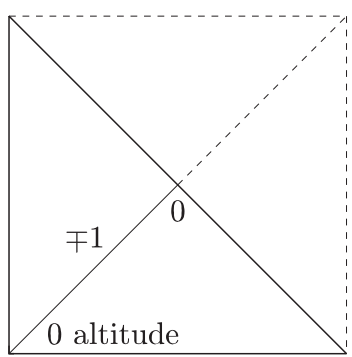

(c)

Fig. 3.1

Let $\Delta_{A B C}$ denote a floor (isosceles right triangle) of the prism. We choose coordinates $A=\{-1,-1\}, B=\{1,-1\}$, and $C=\{-1,1\}$ for the vertices of the triangle; Fig. 3.1(a). Let $f_{1,2}(t)(t \in[-1,1])$ be a parametrization of a closed trajectory of a ball in $\Delta_{A B C}$ disjoint from corners of the triangle. This trajectory can be "unfolded" into the trajectory in the square $\square_{A B C D}=([-1,1] \times[-1,1])$; Fig. 3.1(b), which can be parametrized by 
$\left(f_{1}(t), f_{2}(t)\right)$ where $f_{i}(t)=f\left(n_{i} t+c_{i}\right)$ and $f(t)$ is the "sawtooth" function of Fig. 1.1. If $\left(f_{1,2}(t), f_{3}(t)\right)$ describes a knot in the prism then the associated "unfolded" curve $\left(f_{1}(t), f_{2}(t), f_{3}(t)\right)$ describes a knot in a cube. In particular $n_{1}, n_{2}$, and $n_{3}$ are pairwise relatively prime integers.

THEOREM 3.12. Let $\left(f_{1,2}(t), f_{3}(t)\right)$ describe a knot, $K$, in an isosceles right triangular prism. If $n_{1}$ and $n_{2}$ are odd then:

(i) $n_{3}$ has to be odd, except for the degenerate case desribed in Fig. 3.1(c). Here $n_{3}=2$ and $n_{1}=n_{2}=1$; furthermore, the knot in the triangualar prism is trivial, but the "unfolded knot" in the cube has one singularity (at the point $(0,0, s)$ for some $s$ ).

(ii) $K$ is $Z_{2}$-periodic and the linking number of an axis of the $Z_{2}$-action with the knot is equal to \pm 1 .

Proof. (i) Since $\left(f_{1}, f_{2}\right)(t+1)=-\left(f_{1}, f_{2}\right)(t)$, it follows that if $\left(f_{1}, f_{2}\right)(t)$ is in $\Delta_{A B C}$ then $\left(f_{1}, f_{2}\right)(t+1)$ is in $\Delta_{A D C}$. Thus $f_{1,2}(t+1)$ and $f_{1,2}(t)$ are symmetric with respect to the diagonal $(-1,-1),(1,1)$. Furthermore the above symmetry preserves the orientation of the trajectory (in $\Delta_{A B C}$ ). If $n_{3}$ is even then $f_{3}(t+1)=f_{3}(t)$ and therefore the knot $K$ is symmetric with respect to the plane of the rectangle $[(-1,-1),(0,0)] \times[-1,1]$ and the symmetry preserves the orientation of $K$. The only way for $n_{3}$ to be even is for $K$ to be disjoint from the symmetry plane or fully to lie on the plane. The first case is impossible and the second leads to the degenerate situation described in Theorem 3.12(i).

(ii) If $n_{3}$ is odd then $f_{3}(t+1)=-f_{3}(t)$ and thus the line going through $(-1,-1,0)$ and $(0,0,0)$ is the axis of symmetry for $K$, disjoint from $K$. Therefore $K$ is $Z_{2}$-periodic. To find the linking number of the axis with $K$ we follow the method of the proof of Theorem 3.3 and, in particular, the proof of Lemma 3.5. Our knot is a deformation of the singular closed curve whose projection goes through $(0,0)$, reaches to the corners of the square where it bounces and "bends on itself". A small deformation of the parameters naturally gives the linking number \pm 1 and any deformation omitting $(0,0)$ does not change this linking number.

4. Further speculations. One possibility of extending the family of billiard knots in a cube is to relax the condition on $\mathcal{F}$ from the first section. We no longer require that our trajectories are time-reversible. We will give two examples below, the second motivated by movement in ponds of a unicellular organism Paramecium Caudatum also called Slipper Animalcule.

Definition 4.1. Consider the family $\mathcal{F}^{\prime}$ of continuous fuctions $f: R \rightarrow R$ which satisfy the following properties:

(i) $f(t+1)=-f(t)($ skew-period 1$)$,

(ii) $f(0)=1$ (initial condition),

(iii) $f$ is strictly decreasing on the interval $[0,1]$.

As before, we define $f_{i}(t)=f\left(n_{i}(t)+c_{i}\right)$. We say that a knot is an $\mathcal{F}^{\prime} k n o t$ if it can be parametrized by $\left(f_{1}(t), f_{2}(t), f_{3}(t)\right)$.

As a consequence of our conditions we immediately have the following: 
(iv) $f$ has period $2 ; f(k)=(-1)^{k}$ for any integer $k$.

(v) For any $f \in \mathcal{F}^{\prime}$, there is a unique number $b \in(0,1)$ such that $f(b)=f(b+1)=0$.

(vi) $\mathcal{F}^{\prime}$ is a convex space; that is, if $f, g \in \mathcal{F}^{\prime}$, then $(1-s) f+s g$ is in $\mathcal{F}^{\prime}$ for any $s \in[0,1]$.

We do not know whether every $\mathcal{F}^{\prime}$ knot is a billiard knot but we can extend Theorem 3.2 to this family of knots.

THEOREM 4.2. If $\left(f_{1}(t), f_{2}(t), f_{3}(t)\right)$ parametrize a knot $K$ in $R^{3}$ then:

(i) If all $n_{i}$ 's are odd then $K$ is strongly + amphicheiral.

(ii) If the $n_{i}$ 's are pairwise relatively prime numbers and $n_{3}$ is even then $K$ is a $Z_{2}$-periodic knot and the linking number between the axis of the $Z_{2}$-action and $K$ is equal to \pm 1 .

Proof. (i) We use the condition (i) to get $f_{i}(t+1)=-f_{i}(t)$; thus $K$ is preserved by the central symmetry of $R^{3}$.

(ii) As in the case of the proof of Theorem 3.2, the proof of Theorem 4.2(ii) will be completed if we prove the following lemma.

LEMMA 4.3. If a closed planar curve $\left(f_{1}(t), f_{2}(t)\right)$, for co-prime odd integers $n_{1}, n_{2}$, does not go through $(0,0)$ then the linking number, $l k\left(f_{1}, f_{2}\right)$, of the curve with $(0,0)$ is equal to +1 or -1 .

Pr o of. The closed planar curve $\left(f_{1}(t), f_{2}(t)\right)$ goes through 0 if and only if $n_{1} t+c_{1}=$ $b+h_{1}$ and $n_{2} t+c_{2}=b+h_{2}$ for some integers $h_{1}, h_{2}$. Thus

$$
\frac{c_{2}}{n_{2}}-\frac{c_{1}}{n_{1}}=\frac{h_{2}}{n_{2}}-\frac{h_{1}}{n_{1}}+b\left(\frac{n_{1}-n_{2}}{n_{1} n_{2}}\right) .
$$

One should notice that $h_{1}, h_{2}$ are unique in the sense that if for different $t$ (say $t^{\prime}$ ) one gets $h_{1}^{\prime}, h_{2}^{\prime}$ then $h_{1}^{\prime}=h_{1}+s n_{1}$ and $h_{2}^{\prime}=h_{2}+s n_{2}$ for some integer $s$. Thus $t^{\prime}$ is congruent to $t$ or $t+1$ modulo 2. Without loss of generality we can assume that $c_{1}=0$. Now consider the open annulus parametrized by $c_{2} \in[0,2] /(0 \sim 2), b \in(0,1)$. We are considering all $b$ corresponding to functions $f \in \mathcal{F}^{\prime}, f(b)=0$. The subspace of the annulus for which $\left(f_{1}, f_{2}\right)$ goes through 0 is of codimension 1 . This is a collection of intervals given by the condition $b\left(n_{1}-n_{2}\right)=n_{1} c_{2}-n_{1} h_{2}+n_{2} h_{1}$. Each of the components of the complement of the intervals contains a point with coordinate $b$ equal to $\frac{1}{2}$. Thus the theorem reduces to the case $f\left(\frac{1}{2}\right)=0$, as the linking number for every path-connected region of the annulus is constant. Now consider a function $g$ which satisfies also $g(-t)=g(t)$ (for example $\cos (\pi t))$, and let $g^{s}=s g(t)+(1-s) f(t)$. Of course $g^{s}\left(\frac{1}{2}\right)=0$, thus for given $c_{2}$, if $f(t)$ omits zero then $g^{s}(t)$ also omits zero. Therefore, by homotopy invariance of the linking number and by Lemma 3.5, $l k\left(f_{1}(t), f_{2}(t)\right)=l k\left(g_{1}(t), g_{2}(t)\right)= \pm 1$ as required.

Paramecium Caudatum. Members of the genus Paramecium are unicellular organisms and hence placed in the phylum Protozoa. Paramecium Caudatum is the "slippershaped animalcule" of the early microscopists that is widely distributed and extensively studied. It commonly measures 170-290 microns. The species is world-wide in distribution and commonly found in ponds and bodies of stagnant and fresh water. The Paramecium Caudatum can swim in a straight line. When it strikes a solid object, it backs away and 
tries a new direction [Wi53]. According to J. Dembowski, the angle of reflection off the object is approximately constant.

We will propose two types of trajectories, motivated by movement of Paramecium Caudatum, and so relaxing the condition that the angle of incidence be equal to the angle of reflection.

Definition 4.4. (i) We consider a Paramecium room to be a Cartesian product $M \times N$ of a 2-dimensional Riemannian manifold $M$ (with a piecewise smooth boundary) and a 1-dimensional Riemannian manifold $N$. A Paramecium moves along a geodesic line in $M \times N$ with constant speed until it hits the boundary. Then it reflects in such a way that in a horizontal direction the reflection angle is constant (say $\alpha \leq \pi / 2$ ) when a vertical wall is hit, and the vertical component changes its sign when the floor or ceiling is hit.

(ii) A room of constant reflection angle is a Riemannian manifold $M$ with a piecewise smooth boundary such that a particle moves along a geodesic line in $M$ with constant speed until it hits the boundary. Then it reflects in such a way that it stays on the plane of the vector normal to the boundary and the trajectory before the reflection, and the value of the reflection angle is constant. If the particle hits a corner or hits a boundary along the vector normal to the boundary, its further motion is usually not defined.

The natural setting for Paramecium knots (simple closed trajectories in a Paramecium room) is a polygonal prism. The advantage of the Paramecium knot setting is that the segments of the projection of the trajectory have only a finite number of directions, so the case of irrational polygons is not much more difficult than the rational one. We will end this section by showing that the set of Paramecium knots (with $\alpha=\pi / 4$ ) in rectangular prisms is the same as the set of billiard knots in a cube (or, according to Corollary 1.4, Lissajous knots). Fig. 4.1(b) represents the knot of type $5_{2}$ [Ro76], as a billiard knot in a cube $\left(f_{1}(t)=f\left(2 t-\frac{1}{6}\right), f_{2}(t)=f(3 t), f_{3}(t)=f\left(7 t+\frac{1}{4}\right)\right)$. Fig. 4.1(a) represents the knot $5_{2}$ as a Paramecium knot $(\alpha=\pi / 4)$ in a rectangular prism.

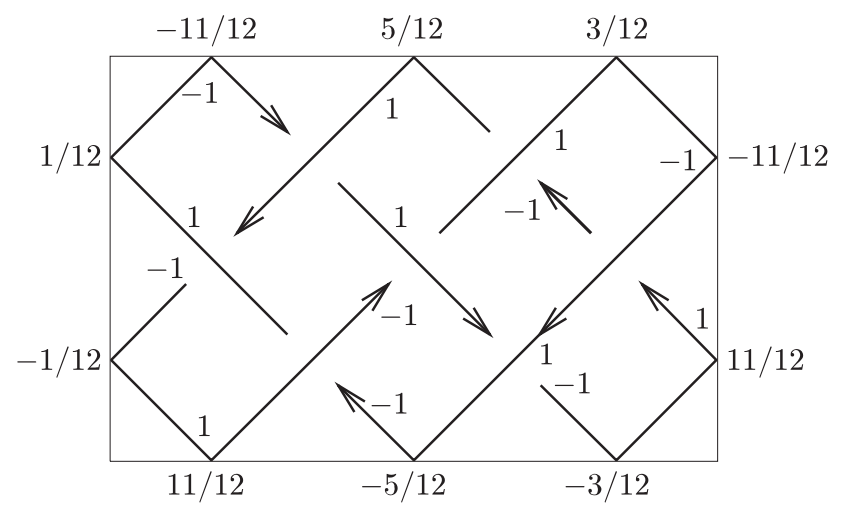

(a)

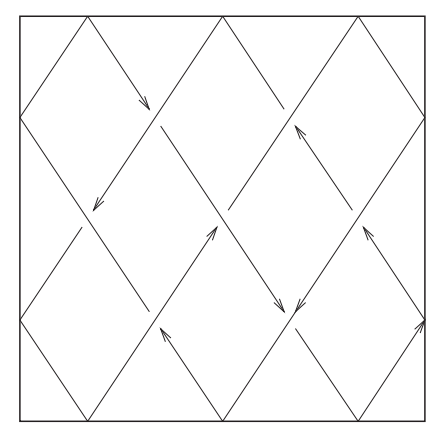

(b)

Fig. 4.1. The knot $5_{2}$.

THEOREM 4.5. There is a bijection between billiard knots (in a cube) of type $n_{1}, n_{2}, n_{3}$ and Paramecium knots in a rectangular prism $\left(\left[-n_{2}, n_{2}\right] \times\left[-n_{1}, n_{1}\right] \times[-1,1]\right)$, for $\alpha=\pi / 4$. 
Proof. Consider the automorphism (homothety) of $R^{3}$ given by $(x, y, z) \rightarrow\left(n_{2} x\right.$, $\left.n_{1} y, z\right)$. Then a billiard trajectory in a cube is sent to a Paramecium trajectory in a rectangular prism (with $\alpha=\pi / 4$ ). The map is a bijection between the trajectories so the proof is complete.

A similar fact also holds for constant reflection angle knots.

THEOREM 4.6. There is a bijection between billiard knots (in a cube) of type $n_{1}, n_{2}, n_{3}$ and constant reflection angle knots in the rectangular prism $\left(\left[-n_{2} n_{3}, n_{2} n_{3}\right] \times\left[-n_{1} n_{3}, n_{1} n_{3}\right]\right.$ $\left.\times\left[-n_{1} n_{2}, n_{1} n_{2}\right]\right)$, for $\alpha=\pi / 4$.

In Figure 4.2, we show an interesting example of a billiard link (also a Paramecium link with $\alpha=\pi / 3$ ) in a regular triangular prism. This 3 -dimensional figure of the link $9_{24}^{2}$ [Ro76] was made by M. Veve. This link is interesting because A. Hatcher and A. Reid [Re91] showed that its complement has a hyperbolic structure and the group of the knot is an arithmetic group (subgroup of $\mathrm{PSL}_{2}\left(\mathrm{O}_{2}\right)$, where $\mathrm{O}_{2}$ is the ring of integers in the imaginary quadratic number field $Q(\sqrt{-2}))$.

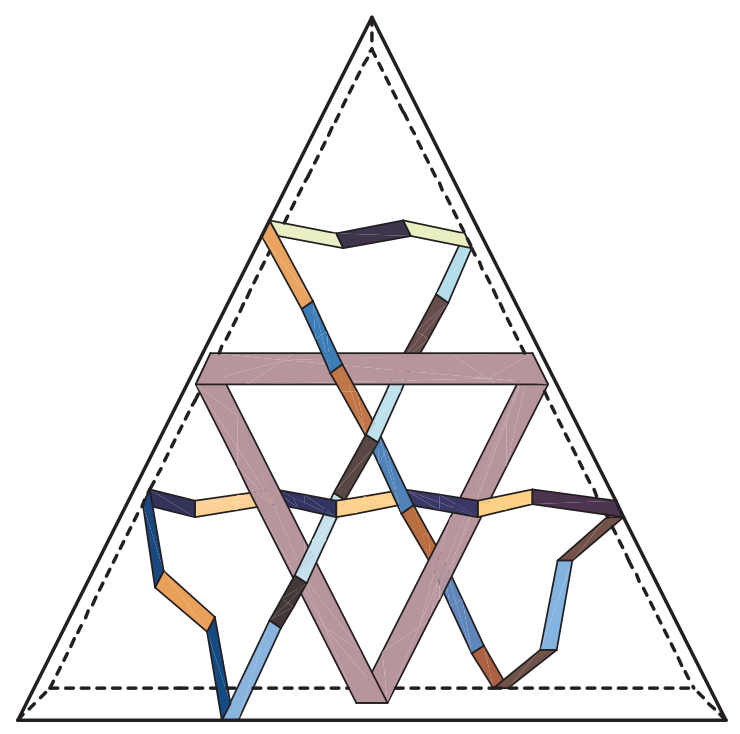

Fig. 4.2. The link $9_{24}^{2}$ as a billiard (also Paramecium) link.

\section{References}

[BHJS94] M. G. V. Bogle, J. E. Hearst, V. F. R. Jones and L. Stoilov, Lissajous knots, Journal of Knot Theory and Its Ramifications 3 (1994), 121-140.

[BGKT94] M. Boshernitzan, G. Galperin, T. Kröger and S. Troubetzkoy, Some remarks on periodic billiard orbits in rational polygons, preprint, SUNY Stony Brook, 1994. 
[Cro95] P. Cromwell, Borromean triangles in Viking art, Math. Int. 17 (1995), 3-4.

[GKT94] G. Galperin, T. Kröger and S. Troubetzkoy, Local instability of orbits in polygonal and polyhedral billiards, Comm. Math. Phys. (1994).

[GSV92] G. Galperin, A. Stepin and Ya. B. Vorobets, Periodic billiard trajectories in polygons: generation mechanisms, Russian Math. Surveys 47 (3) (1992), 5-80.

[HK79] R. Hartley and A. Kawauchi, Polynomials of amphicheiral knots, Math. Ann. 243 (1979).

[KMS86] S. Kerckhoff, H. Masur and J. Smillie, Ergodicity of billiard flows and quadratic differentials, Ann. of Math. 124 (1986), 293-311.

[Mu71] K. Murasugi, On periodic knots, Comment. Math. Helv. 46 (1971), 162-174.

[Pr95] J. H. Przytycki, Symmetry of knots: Lissajous and billiard knots, notes from a talk given at the Subfactor Seminar, U.C. Berkeley, May 1995.

[Re91] A. W. Reid, Arithmeticity of knot complements, J. London Math. Soc. (2) 43 (1991), 171-184.

[Ro76] D. Rolfsen, Knots and links, Publish or Perish, 1976.

[Ta95] S. Tabachnikov, Billiards. The Survey, preprint, 1995.

[Wi53] R. Wichterman, The biology of Paramecium, The Blakiston Company, Inc., New York, Toronto, 1953. 